\title{
Theoretical calculation of nitro-1-(2,4,6-trinitrophenyl)-1H-azoles energetic compounds
}

\author{
Zhibin Qi ${ }^{1}$, Yong $\mathrm{Lu}^{1}$, Rui-Jun Gou ${ }^{1}$, and Shu-Hai Zhang ${ }^{1}$ \\ ${ }^{1}$ North University of China
}

January 5, 2022

\begin{abstract}
In order to study the properties of new energetic compounds formed by introducing nitroazoles into 2,4,6-trinitrobezene, the density, heat of formation and detonation properties of 36 nitro-1-(2,4,6-trinitrobenzene)-1H-azoles energetic compounds are studied by density functional theory, and their stability and melting point are predicted. The results show that most of target compounds have good detonation properties and stability. And it is found that nitro-1-(2,4,6-Trinitrophenyl)- 1 H-pyrrole compounds and nitro-1-(2,4,6-trinitropenyl)-1H-Imidazole compounds have good thermal stability, and their weakest bond is $\mathrm{C}-\mathrm{NO} 2$ bond, the bond dissociation energy of the weakest bond is $222 \mathrm{~kJ}$ mol-1-238 kJ mol-1 and close to TNT (235 kJ mol-1). The weakest bond of the other compounds may be the C-NO2 bond or the N-N bond, and the strength of the N-N bond is related to the nitro group on azole ring.
\end{abstract}

\section{Hosted file}

Theoretical calculation of nitro-1-(2,4,6-trinitrophenyl)-1H-azoles energetic compounds.docx available at https://authorea.com/users/454105/articles/551840-theoretical-calculation-ofnitro-1-2-4-6-trinitrophenyl-1h-azoles-energetic-compounds 